\title{
First left main coronary revascularization with adjuvant intracoronary lithotripsy in Spain
}

\author{
Revascularización del tronco coronario distal apoyada en sistema de litoplastia \\ coronaria: experiencia inicial en España
}

\author{
Raymundo Ocaranza-Sánchez*, Rosa A. Abellás-Sequeiros, and Carlos González-Juanatey \\ Interventional Cardiology Unit, Hospital Universitario Lucus Augusti, Lugo, Spain
}

\section{Clinical case}

We present the case of an 83-year-old male patient, former smoker and dyslipidemic, with a history of ischemic heart disease treated with percutaneous revascularization (PR) on middle anterior descending (AD) and proximal circumflex (Cx) arteries in 2001. He was admitted to the authors' center intensive care unit with a diagnosis of non-ST-segment elevation (NSTE) acute coronary syndrome of the NSTE acute myocardial infarction type. Admission electrocardiogram showed no acute repolarization alterations and the echocardiogram documented a preserved biventricular systolic function without segmental contractility alterations or significant valvulopathies. Diagnostic coronary angiography showed $85 \%$ severe lesion with abundant calcium in the left coronary trunk (LCT) that affected the $A D$ and $C X$ ostia, with Medina of 1-1-1 (Scheme 1, Fig. 1A and B). Stents implanted on $A D$ and $C x$, without restenosis

After a medical-surgical session, PR on the distal LCT lesion was decided. The large calcium component of the stenosing plaque forced to plan the intervention based on an exhaustive modification of the initial plate. Rotational atherectomy was not considered to be the first-line technique in this case due to the discrepancy between the vessel reference diameter and the available diameters of rotablation olive-shaped burrs. In this context, plaque modification was decided with the Shockwave ${ }^{\circledR}$ Coronary Lithoplasty System.

Initially, severe angulation and stenosis of the lesion to be treated allowed only the passage of the introducer sheath toward the distal AD. The Shockwave ${ }^{\circledR}$ Lithoplasty System was advanced over it and crossing of the distal LTC lesion toward ostial DA was achieved. Keeping the device insufflated at $4 \mathrm{am}$, a first 10-wave pulse was applied (with incomplete lesion expansion, Fig. 1C, arrow), followed by four applications more of 10 pulses each, with a Lithoplasty balloon at 6 atm. As a result, an adequate expansion of the balloon was achieved (Fig. 1D), with partial opening of the lesion. This allowed the passage of the guidewire to the distal $\mathrm{Cx}$ and $\mathrm{Cx}$ ostium predilation with scoring balloons of increasing diameters (Fig. 1E and F). Then, the Lithoplasty balloon was advanced toward the ostial Cx (Fig. 1G) and 20 pulses, at a nominal pressure of 4 atm, were applied, with an increase of vessel permeability at that level (Fig. 1H).

In view of the degree of disease and severe angulation at the exit of both coronary ostia (AD and CX), an LCT revascularization with double stent (T-stent) was decided. Thus, the first drug-eluting $3.5 \mathrm{~mm}$ stent was implanted above the proximal $\mathrm{Cx}$ adjusted to the ostium and the second $3.5 \mathrm{~mm}$ everolimus stent on LCT-AD
Correspondence:

*Raymundo Ocaranza-Sánchez

E-mail: raymundoocaranza@yahoo.com 2604-7063/@ 2019 Instituto Nacional de Cardiología Ignacio Chávez. Published by Permanyer. This is an open access article under the CC BY-NC-ND license (http://creativecommons.org/licenses/by-nc-nd/4.0/) 


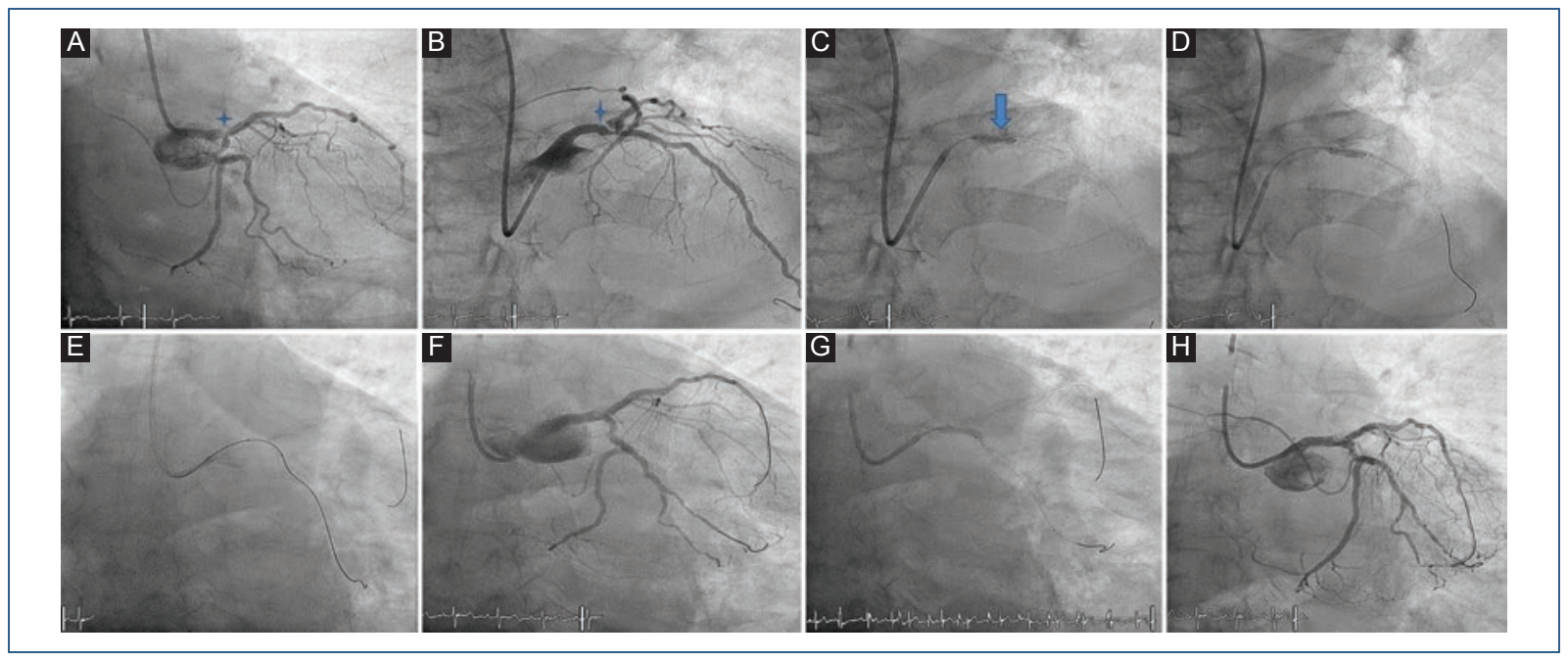

Figure 1. Percutaneous coronary revascularization of the left coronary trunk with coronary Lithoplasty. A and B: baseline angiography; C-G: Lithoplasty balloon and stent implantation; H: final result.

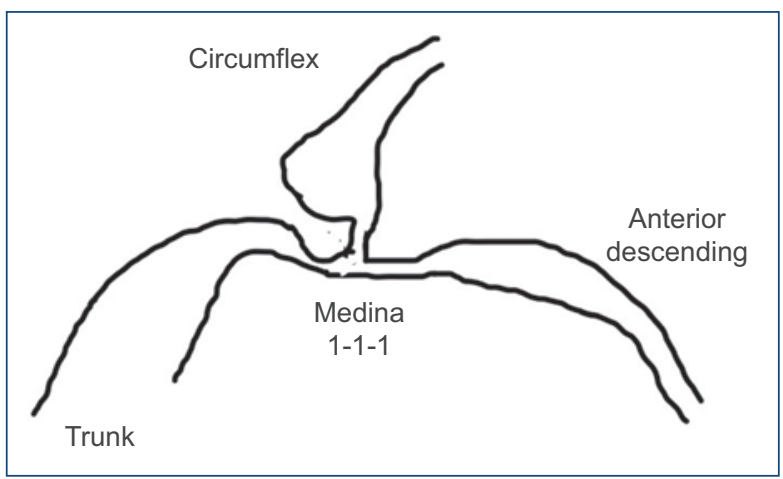

Scheme 1. Graphic representation of distal trunk lesion affecting $\mathrm{AD}$ and CX ostia.

was released. The final result was optimized using the POT technique with post-dilation with non-distensible balloons on LCT (Fig. 2A and B), with adequate expansion and apposition of the stent on LCT being checked by IVUS (Fig. 2C and D). The patient was discharged under dual antiplatelet therapy with ASA and clopidogrel, without further incidents.

\section{Discussion}

The first experience in Spain of LCT PR supported by Shockwave ${ }^{\circledR}$ plaque modification system is presented. Said technology is based on the use of sonic waves that, emitted in low-frequency pulses, allow the destructuring of calcium in the intima and middle layers of the arterial wall, without affecting soft tissues, by fracturing

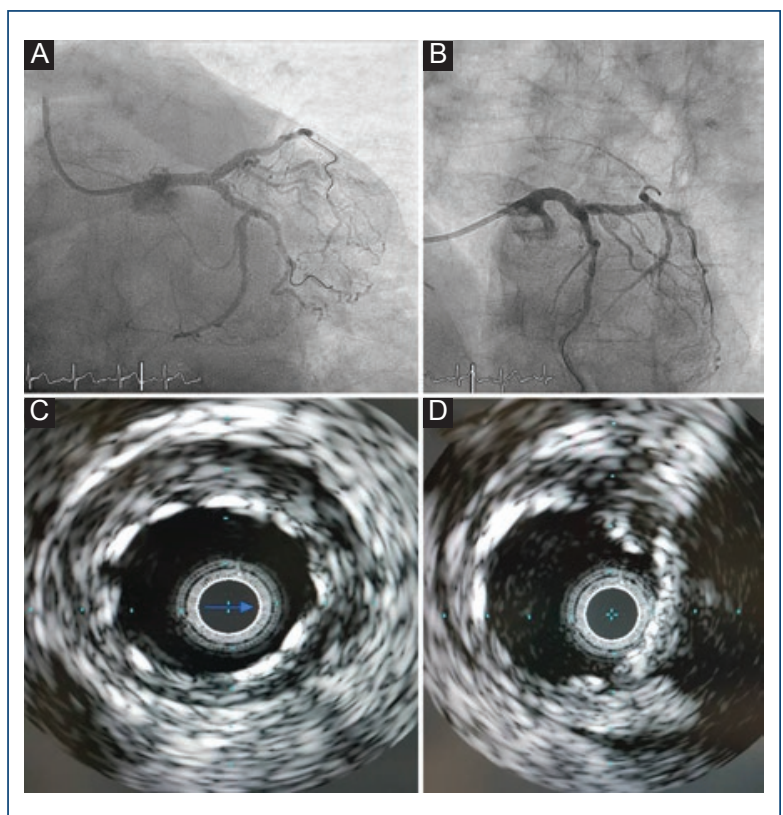

Figure 2. Final angiographic and IVUS result after LCT revascularization. A and B: final angiographic result; C and D: IVUS images showing stent adequate expansion and apposition.

the plaque and making it dilatable. The device consists of an angioplasty balloon with rapid diameter exchange from 2.5 to $4 \mathrm{~mm}$ and a fixed length of $12 \mathrm{~mm}$, which contains within two sensors that release the acoustic signal. These waves, in contact with the saline medium filling the balloon, generate an expansion wave that perpetuates itself on calcium and gives rise to its 
modification, after which the stent implantation can be done without the risk of underexpansion. The crossing profile of the balloon allows its use through $6 \mathrm{~F}$ guiding catheters; as in this case, radial access can be the approach of choice with this device. On the other hand, the simplicity of its use is an important point in comparison with other plaque modification devices, such as atherectomy in its different versions. According to the primary study of the DISRUPT CAD II device ${ }^{1}$, this procedure does not produce acute complications, such as perforation, acute occlusion, or no-flow or slow-flow phenomena ${ }^{1,2}$. In addition, higher performance is observed, with higher levels of fracture and plaque modification in cases where the calcium load is larger and circumferential, which reduces the eccentricity index after stent implantation, with better symmetry of the final vessel2,3.

This case supports the efficacy and safety of this new technique for high calcium content plaque modification in a highly complex scenario, such as LCT bifurcation.

\section{Funding}

No funding was received for this study.

\section{Conflicts of interest}

There are no conflicts of interest.

\section{Ethical disclosures}

Protection of people and animal subjects. The authors declare that no experiments were performed on humans or animals for this study.

Confidentiality of data. The authors declare that no patient data appear in this article.

Right to privacy and informed consent. The authors declare that no patient data appear in this article.

\section{References}

1. Brinton T, Hill J, Ali Z. DISRUPT CAD: a multicenter, prospective, single-arm study of percutaneous lithoplasty prior to stent implantation in heavily calcified coronary lesion. J Am Coll Cardiol. 2016;68 TCT Suppl. Available at: https://www.tctmd.com/slide/disrupt-cad-multicenterprospective-single-arm-study-percutaneouslithoplasty-prior-stent. Accessed July 13, 2017.

2. Ali ZA, Brinton T, Hill JM, Maehara A, Matsumura M, Karimi Galougahi K, et al. Optical coherence tomography characterization of coronary lithoplasty for treatment of calcified lesions: first description. JACC Cardiovasc Imaging. 2017:10 (8):897-906.

3. Serruys PW, Katagiri Y, Onuma Y. Shaking and breaking calcified plaque: lithoplasty, a breakthrough in interventional armamentarium? JACC Cardiovasc Imaging. 2017;10(8):907-11. 